\title{
Index rerum ad Vol. 12
}

Confecit Wilhelm Baumann, Zurich

Acid-fast bacilluria, 6 Adenoma prostatae, biopsia, 1 Adrenal cortex, hyperactivity and loss of sexual function, 48 Anaesthesia et fonctio renalis, 228 Anomalia de riñón y de vesícula seminal,

49

Bacilluria, Mycobacteriuria, 6 Bâtonnets acido-résistants, 6 Biopsia prostatica percutanea, 1 Bladder, carcinoma, diagnostic, application of P 32, 63 -, -, radioisotopic treatment, 63 -, neck contracture, transurethral resection, 122 neoplasm, Phenolphthalein test, 328

papillomatosis, diagnostic application of $\mathrm{P} 32,63$ radioisotopic treatment, 63

pedunculated papilloma, 26

radioisotopic treatment of tumors, 63

specific ulcerous cystitis, 358

- $\quad$ tumor, transurethral resection, 122

Blutungen, intra- und postoperative, 337

Cancer vésical, test à la Phénolphthaline,

328 Carcinoma prostatae, biopsia, 1 , resectio transurethralis, 122

Citodiagnostica in urologia, 307

Coagulation-system, 337

Colic attack, influence of renal function,

204 Cryptorchism, vesiculography, 289 Cystitis ulcerosa specifica, 358 Cystogramm, 254

Cystometrogramm, 254 Cytological diagnosis in urology, 307

Diodrast, radioisotope renogramm, 148

Emorragie intra e post-operatorie, 337 Epididymographia, 289 Epidural anesthesia and renal function, 228

Ethylurethane and renal function, 228 Eunuchoidísm, gonadotropin test, 135 Extrait prostatique, examen cytolo-gique, 307

Fonction sexuelle en cas de lesion de la moelle, 270 Franklin-Vim-Silvermann biopsy needle, 1

Gonadotropin test, 135 Guajazulen, 358

Harnblase, Behandlung des Papilloms, 26

-, Carcinom, diagnostische Verwen-dung von P 32, 64

-, Innervation, 254

-, Miktionsmechanismus, 254

-, neurogene Reizblase, 254

-Papillomatose, Behandlung mit Radioisotopen, 63 
63

, diagnostische Applikation von P32,

-, Radioisotopenbchandlung von Tumo-ren, 63

-, spezifische ulceröse Cystitis, 358

-, Phenolphthaleintest, 328

Hemorrhage, operative and postoperative, 337

Hochdruck renalen Ursprungs, 191,219

Hodenbiopsie bei Rückenmarksverletz-ten, 270

Horse shoe kidney, venogram, 280

Hutter symptom, 146

Hydronephrosis, radioisotope renogramm, 148

-, venogram, 280

Hypercorticismus, impotentia coeundi, 38

Hypernephrom und Hyperplasie der Prostata, 38

Hypernephroma, venogram, 280

Hypertension of renal origin, 191, 219

Hypertension, radioisotope renogramm, 148

Hypothermia renalis, 161

ХП/1961

Index rerum

371

Hypertrophia prostatica, resectio trans-

urethralis, 122 Hypogonadism, gonadotropin test, 135

Impotentia coeundi, Hypercorticismus,

38 Ipercortico-surrenalismo, perdita della

funzione sessuale, 38 Ipertensione di origine renale, 191, 219 Ipossia da occlusione dei vasi renali, 228 Ipotermia, renale, 161 Ischaemia renalis, 161 Isotopi radioattivi, trattamento del tumore vesicale, 63 Isotopos radioactivos, renograma, 148

17-Keto-Steroide bei Rückenmarksver-

letzten, 270 Kidney, cytological diagnosis, 307

ectopic, 164, 246

effect of epidural anesthesia, 228

effect of urethane derivates, 228 -, hypernephroma, venogram, 280

hypertension, 191, 219

hypothermia, 161

hypoxia, 228

influence of colic attack, 204 -, ischemia, 161

malformation, 49 , venogram, 280

nephroptosis, 164, 246

-, radioisotope renogramm, 148 -, renal venogram, 280

- $\quad$ tuberculosis, 191

-, radioisotope renogramm, 148

L-eydig's cells, gonadotropin test, 135 Liquido prostatico, istologia, 307 Lithiase urétérale, rénogramme radio-isotopique, 148

Malformations of the kidney, venogram, 
49, 280 Micturition, mechanism, 254 Mißbildung von Nieren und Samen-

blasen, 49, 280 Mycobacteriuria, 6

Neoplasie vesicale, test con la Fenolftaleina, 328

Nephrectomy in hypertony, 219

Nephrolithiasis, radioisotope renogramm, 148

-, renal function, 204

Nephropexis, 164, 246

Nephropexy, free skin flap method, 246

Nephroptosis, 164, 246

Neurosis prostatica debido a hiper-corticalismo, 38

Névrose prostatique et hyperfonction

surrénalienne, 38 Niere, Cytologie bei Tumoren, 307 -, Einfluß der Ureterkolik, 204 -, Ektopie, 164, 246 -, funktionelle Störungen, Radioisoto-

pen-Renogramm, 148 -, Hypertonie, 191, 219 -, Hypothermie, 161 -, Hypoxie bei

Gefäßverschluß, 228 -, Ischämie, 161

- $\quad$ Nephroptose, 164, 246

-, radioisotope Rénogramme, 148 -, Tuberkulose, 191

- Venogramm bei Mißbildungen, 280

Nierentuberkulose, Venogramm, 280

Octylurethane and renal function, 228

Papilloma vesicae, therapia, 26, 63 Papillomas vesicales pediculados, trata-

miento, 26 Pelvic tomors, radioisotopic treatment,

Ill Pelvis renalis, hypotonia, 164 Phenolphthalein tolerance test, 328 Prostata, biopsia percutanea,

1 Prostata, carcinoma, transurethral resection, 122 -, hypertrophy, transurethral resection,

122 Prostata, Hyperplasie bei Hyperne-

phrom, 38 Prostataexprimat, Cytologie, 307 Prostatic hyperplasia, vesiculography, 289

, hypernephroma, 38

, transurethral resection, 122

- $\quad$ neurosis, 38

Prostectomy, disturbances of blood coagulation, 337 Psoasedge symptom, 164 Psoasrand-

Symptom, 164 Pyelography in nephroptosis, 164, 246

Narath symptom, 164 Neurogenic bladder, 254 Radioisotope renogramm, 148 Radioisotopic

treatment, experimental

studies, Ill

, bladder tumors, 63

Raggi X, trattamento dei tumori pel-

vici, Ill Rein, diagnose cytologique, 307

- $\quad$ ectopique, 164,246

-, vésicules séminales, malformations, 49 -, hypertonie, 191, 219 -, hypothermie, 161 -, hypoxie

228

- $\quad$ influence de la colique, 204

372

Index rerum

XII/1961

Rein, ischémie, 161 
-, l'effet de l'Uréthane, 228

-, Гeffet de Гanesthésie épidurale, 228

$\sim$, lithiasique et fonction rénale, 204

-, néphroptose, 164, 246

-, rénogramme radioisotopique, 148

- $\quad$ tuberculeux, 191

-, vénogramme, 280

Renal pelvis, hypotony, 164

Renogramm, radioisotope, 148

Resectio transurethralis, 122

Riñón ectópico, 164, 246

Rückenmarksverletzte, Sexualfunktion,

270 Samenblasendarstellung bei Rücken-

marksverletzten, 270 Samenblasen, Mißbildung, 49 Säurefeste Stäbchen im Urin, 6 Secréción

prostatica, histologia, 307 Seminal vesicle, malformation, 49 Seminal vesiculography in spinal cord

injury, 270 Sexual function in spinal cord injury, 270 Signo de Narath, 164

- $\quad$ del borde del psoas, 164

Smegma-Bakterien, 6

Spinal cord injury, sexual function, 270 Stuart-Prower factor, 337

Teleterapia, tratamiento de tumores

pélvicos, Ill Test gonadotropinico, 135 Testicular aplasia, vesiculography, 289

biopsy in spinal cord injury, 270

function, gonadotropin test, 135 Transurethral resection, 122 Tuberculose urogénital, bacillurie,

6 Tuberculosi del rene sinistro, renogram-

ma mediante radioisotopi, 148 Tuberculosis, Mycobacteriuria, 6

urogenitalis, 191

-, bacilluria, 6

, venogramm, 280

, vesiculographia, 289

- $\quad$ vesicae urinariae, 358

Tumeurs pelviennes, traitement par

rayons-X, Ill

- $\quad$-, traitement radioisotopique, Ill

Ureterkolik und Nierenfunktion, 204

Urethane derivates and renal function, 228

Urethra, female, Roentgen examination, 30

Urinary bladder, innervation, 254

, neurogenic, 254

Urogenitaltuberkulose, 148, 191, 289

-, Mykobakteriurie, 6

Urogénital tuberculosis, radioisotope renogramm, 148

Urogénital tuberculosis, vesiculography, 289

Venogram, renal, 280 Vesica urinaria, cystitis ulcerosa spaci-fica, 358

- $\quad$-, innervatio, 254

Vésicules seminales, malformations, 49 Vesiculitis chronica, vesiculographia, 
289 Vesiculography, seminal, 289 Vessie, cancer, application diagnostique du P 32, 63

, traitement radioisotopique, 63

-, etudes sur Гinnervation, 254 -, mécanisme de la miction, 254 -, neurogène, 254

- $\quad$ papillomatose, application diagnos

tique du P 32, 63

, traitement radioisotopique, 63

-, traitement des tumeurs pédiculées, 26

- $\quad$ radioisotopique des tumeurs, 63

X-ray, treatment of pelvic tumors, Ill 